\title{
Phenotypic and genotypic features of strains of Actinobaculum suis- the causative agent of urinary tract infections in pigs
}

\author{
V.I. Pleshakova*, N.A. Lescheva, T.I. Lorengel, and A.V. Konev \\ Federal State Budgetary Educational Institution of Higher Education "Omsk State Agrarian \\ University named after P.A. Stolypin", Omsk, Russian Federation
}

\begin{abstract}
UTI) are a significant problem of infectious pathology among the pig population. Many researchers consider UTI in pigs as a result of the influence of conditionally pathogenic microorganisms and unfavorable conditions of keeping and feeding. In the middle of the last century, a number of researchers identified a specific pathogen, namely Actinobaculum suis, which causes a pathological condition of the bladder and kidneys in pigs. The microorganism remains poorly studied. In morphometric and biochemical terms, it has a weak variance of phenotypic traits, with the greatest fluctuations in the enzyme profiles of the isolated cultures. It is shown that the size of microbial bodies has a direct dependence not only on the component composition of the growth medium, but also on the native pathological material from which the microorganism was isolated. Molecular genetic studies have shown that the content of GC-pairs was on average $55.5 \mathrm{~mole} \%$, which corresponds to the taxonomic position of the pathogen. At the same time, 16SpRNA restriction analysis did not reveal differences in PCR products, which in turn indicates a high degree of genetic relation of the isolated Actinobaculum suis cultures.
\end{abstract}

\section{Introduction}

In the last three decades, the global livestock industry has been experiencing ambiguous, poorly predicted changes in the nosological profile of infectious pathology [14]. The so-called "old" or classic diseases everywhere fade into the background, or in the view of well-applied specific prevention and general technological and veterinary-sanitary measures disappear completely, often occurring in a mild, asymptomatic form $[1,4]$. At the same time, in animal husbandry, and in particular in industrial pig breeding, infections caused by microorganisms that were previously considered conditionally (potentially) pathogenic began to prevail [4-6]. These include staphylococci, streptococci, escherichia, pasteurella, protea, and a number of anaerobic bacteria $[1,2,7,8]$. In this regard, ignoring the essential role of microorganisms isolated from various animal biotopes, which are

\footnotetext{
*Corresponding author: vi.pleshakova@omgau.org
} 
subject to significant phenotypic and genotypic changes under the influence of biotic and abiotic factors, as a rule, leads to significant economic losses associated with the culling of breeding animals or their death, as well as a decrease in the quantitative and qualitative indicators of animal products [2,3]. The above-mentioned microorganisms often serve as causative agents of urinary tract infections in pigs [4,5]. According to a number of authors in industrial pig farms, diseases of the urinary tract of sows significantly reduce the reproductive potential of breeding stock, and also lead to the culling of about $11-13 \%$ of animals $[7,9,10]$. It should be noted that most researchers assign the leading role in the pathogenesis of urinary tract infections to the above-mentioned conditionally pathogenic microorganisms. Meanwhile, back in the middle of the last century, the English authors Soltys, M. et al. [11] isolated a diphteroid-like bacterium from pig urine samples with characteristic symptoms of bladder and kidneys inflammation and classified it as Corynebacterium polimorhum suis. Later, a number of authors [7, 9, 10, 12] showed the connection of this diphteroid microorganism with the occurrence and development of urocystitis and pyelonephritis in pigs. By the end of the XX century, the causative agent of specific urocystitis and pyelonephritis of sows was isolated in many countries of the world with developed industrial pig breeding [4, 8, 10, 13-15].

In the future, the taxonomic position of the causative agent of urocystitis and pyelonephritis of pigs has repeatedly changed. Thus, in 1992 it was reclassified to the genus Eubacterium, then in 1997 it was assigned to the genus Actinomyces [15]. Currently, due to the new reclassification, it is assigned to the genus Actinobaculum and the species Actinobaculum suis.

Taking into account the presented data on the degree of issue study, the purpose of the presented research is to study a number of phenotypic and genotypic characteristics of Actinobaculum suis cultures isolated from pig population of livestock farms in the Omsk Irtysh region.

\section{Materials and Methods}

The pathological material for the research was urine from sows with clinical symptoms of urinary tract lesions. Urine samples were obtained from animals using a special catheter, and urine was collected during spontaneous urination.

Urine from the forcedly killed and dead animals was obtained from the bladder content, using a catheter. In addition, preputial fluid, urine, and semen obtained from the breeding boars were used as the material. Samples of preputial fluid from the diverticulum of boarsproducers were taken using swab (sterile cotton swabs) directly from the preputial sheath (diverticulum).

Samples of seminal fluid from producers were obtained in farms during the procedure of artificial insemination right after the ejaculate collection.

These samples were taken in pig farms of the Omsk region in the period from 2019 to 2020. For bacteriological studies, Columbian agar was used, which was prepared according to the following recipe: to $1000 \mathrm{ml}$ of distilled water, $40.0 \mathrm{~g}$ of Columbian agar was added, as well as $1.6 \mathrm{~g}$ of agar-agar. The resulting mixture was boiled until the specified ingredients were completely dissolved. After that, the culture medium was sterilized. Then, the above solution was cooled to room temperature and $50 \mathrm{ml}$ of defibrinated horse blood and antimicrobial ingredients were added to it, in particular, $0.01 \mathrm{~g}$ of colistin sulfate, 0.05 $\mathrm{g}$ of metronidazole, $0.015 \mathrm{~g}$ of nalidixic acid. Glucose-blood agar was used as a dense selective nutrient medium. The plantings were cultivated in a thermostat at a temperature of $37^{\circ} \mathrm{C}$. To create anaerobic conditions for the cultivation of isolated microorganisms, Oxoid Ltd., England was used. BBL test strips were used to control the anaerobic parameters in 
the anaerostat. In cultures isolated from various pathological materials of sows and boars, morpho-tinctorial characteristics were studied by Gram staining the preparation smears with methylene blue, and carbolic fuchsin. The presence of acid and alcohol resistance was tested using the Ziehl-Nielsen method, the presence of spores - according to SchaefferFulton. Metachromatic granularity (volutin) was determined using the Neisser method. The biochemical characteristics of the isolated cultures were studied during the cultivation of A. suis isolates using semi-liquid Giss nutrient media with a set of various complex carbohydrates, namely, glucose, arabinose, galactose, maltose, sorbitol, dulcite, ribose, raffinose, xylose, sucrose. To determine the catalase activity of isolated A. suis cultures, a peroxidase test was used. At the same time, a $3 \%$ solution of $\mathrm{H}_{2} \mathrm{O}_{2}$ was applied to the grown colonies of A. suis. The starch hydrolysis reaction was carried out by adding it to the meat-peptone agar. The production of urease by isolated A. suis cultures was determined by the Sachs sample. The proteolytic activity was determined by incubating cultures of microorganisms in meat-peptone gelatin. To study the formation of indole and hydrogen sulfide by A. suis cultures, test strips impregnated with a $12 \%$ solution of oxalic acid and a solution of lead acetic acid were used.

The enzyme profile of the isolated microorganisms was studied using the RapID ${ }^{\mathrm{TM}} \mathrm{SS} / \mathrm{u}$ system. The synergistic effect of the cultures was determined using the CAMP test. The hemaagglutinating properties of the isolated cultures were also determined.

Resistance and/or sensitivity of isolates of the causative agent of urocystitis and pyelonephritis of pigs to a number of antibiotics, nitrofuran and sulfonamide preparations was determined using standard disk test systems.

The sequence of the nucleotide composition of deoxyribonucleic acid was studied by the method of thermal denaturation with a heating rate of $0.5^{\circ} \mathrm{C} / \mathrm{min}$.

The GC pairs content was calculated using the formula $(\mathrm{G}+\mathrm{C})=2.08 \mathrm{~T}_{\mathrm{pl}}-106.4$. To standardize genotypic studies, Escherichia coli deoxyribonucleic acid with a known nucleotide composition was used. Restriction analysis of chromosomal DNA in combination with a gene radioactive probe was also used for genetic studies.

\section{Results and Discussion}

When studying the morphological properties of A. suis cultures isolated from various pathological materials of pigs of different sex and age groups, it was revealed that they are characterized by a relative variation of cytostructural characteristics, as reported by a number of researchers $[11,15]$.

It was found that microscopy of microbiological preparations obtained from smearsprints of inflamed areas of bladder mucous membrane in pigs and the contents of the preputial sheath of boars revealed that out of the entire structural diversity of Actinobaculum suis cells, rod-shaped forms dominated in quantitative terms (86.4\%), while ovoid forms were present in a slightly smaller number (12.2\%). Coccoid microorganisms were present in the smallest amounts $(1.3 \%)$.

It should be noted that the cytomorphometric studies conducted allow to state that the size of bacterial cells of Actinobaculum suis can have relatively wide limits of variation, which is reflected in many works of a number of researchers $[8,11,12]$. For example, it was found that the length of rod-shaped microbial cells is in the range from 1.5 to 3.0 microns, and the width can vary from 0.3 to 0.7 microns. In the ovoid A. suis bacteria, the length is from 1.0 to 2.0 microns, and the width is from 0.3 to 0.5 microns. It should be noted that during incubation of microbial cells of urocystitis and pyelonephritis causative agent in selective agar for 5-6 days, relatively large bacterial cells (5.2 microns in length and 1.2 
microns in width) were often detected.

The cytomorphological studies carried out will allow to establish that the size of bacterial cells of Actinobaculum suis is directly dependent on both the biotope from which the pathogen was isolated and the composition of the nutrient medium.

It was found that the largest sizes were determined in A. suis bacteria isolated from pathological foci of the urinary system (bladder, renal pelvis). At the same time, the A. suis bacteria cultured on selective blood agar had the smallest size. Similar growth characteristics of A. suis cultures are indicated by Soltys et al., 1957. Regardless of the location of isolation source, the bacterial cells of A. suis were Gram-positive, and only in old cultures (7-14 days) single gram-negative cells can appear. The same phenomenon is pointed out by Jones, 1987. In most cases, rod-shaped bacterial cells of the causative agent of urocystitis and pyelonephritis in pigs are colored evenly, while ovoid forms were characterized by weak bipolarity. It should be noted that in older cultures, bipolarity is more pronounced. When cultures of isolated A. suis microorganisms are stained, metachromatic granularity is registered in almost all bacterial cells. Microscopy revealed that the volutin grains in all cases had a blue-purple hue, and the bacterial cells were colored yellow, sometimes light brown. This pattern is noted by a number of researchers $[8,9]$. The cultures isolated by us were not acid-resistant, were immobile, and did not form spores. It should be noted that some authors, in particular Soltys et al. (1957); Larsen (1970), indicate that the biochemical characteristics of the cultures of the causative agent of urocystitis and pyelonephritis in pigs have a certain constancy. At the same time, a number of authors point to their variability in terms of biochemical characteristics $[9,10,11,12]$.

Our studies have shown that A. suis cultures isolated from various pig biotopes in $92.5 \%$ split xylose, in $93.2 \%$ - fructose and $95.1 \%$ - arabinose.

It should be noted that sucrose with the formation of acid without gas was fermented by $32.6 \%$ of A. suis cultures, glucose $-4.3 \%$, and lactose $-2.4 \%$. In addition, the conducted studies have shown that for isolated cultures of A. suis, the characteristic biochemical properties are the following:

- split to acid without the formation of maltose, ribose, xylose gas;

- high urease activity;

- negative catalase.

Most researchers believe that the peculiarities of the course of enzyme interactions to a certain extent determine the phenotypic differences between bacteria [4]. Biochemical studies have shown that $81.3 \%$ of $\mathrm{A}$. suis cultures produced $\beta$-glucosidase. It was also found that $100 \%$ of isolated cultures of the pathogen produced $\alpha$-galactosidase, argenine dehydrolase, $\beta$-galactosidase and D-carboxylase. In addition, $93.2 \%$ of A. suis cultures showed hemolytic activity when co-cultured with S. aureus. The latter, in our opinion, indicates a synergistic effect of the proteins of A. suis on S. aureus bacteria, which in turn leads to the activation of the beta-toxin.

Taking into account the results obtained, it can be stated that A. suis cultures isolated from various pig biotopes have a number of permanent biochemical characteristics that make it possible to identify the isolated microorganisms as Actinobaculum suis. In addition to the above, it should be noted that some A. suis cultures were distinguished by a certain variability of enzyme profiles and biochemical properties.

An important factor in the existence and manifestation of the characteristic phenotypic properties of microorganisms is their persistent properties. One of the important indicators of microorganisms is the ability to exhibit hemagglutinating properties. Studies have shown that $50 \%$ of A. suis cultures agglutinated the red blood cells of the animals included in the testing (pigs, calves, guinea pigs, chickens, horses). At the same time, 1 culture did not agglutinate red blood cells from any animal. 
It was found that the cultures of the causative agent of urocystitis and pyelonephritis in pigs were sensitive to penicillin, levomycitin, oxacillin, ampicillin, trimetaprim and the same time showed resistance to neomycin, sulfalene, kanamycin, norsulfazol, streptomycin.

The analysis of domestic and foreign literature has shown that the comparison of even a large number of phenotypic characteristics of a particular microorganism in most cases does not allow to obtain reliable data that allow to assign microorganisms to certain taxonomic groups $[4,6,8,10]$. At the same time, the use of modern molecular genetic methods makes it possible to clearly differentiate the existing similarities and differences within not only the species, but also individual strains of microorganisms.

To this end, we conducted comprehensive molecular genetic studies, where the first stage was to study the percentage of the total content of guanine and cytosine $(\% \mathrm{G}+\mathrm{C})$ in Actinobaculum suis isolates isolated in 11 pig farms of the Omsk Irtysh region (Table 1).

Table 1. Indicators of the molecular composition of A. suis DNA isolated in pig farms of the West Siberian region

\begin{tabular}{|c|c|c|}
\hline \multirow{2}{*}{ Culture } & Content mole\%, G+C & $\begin{array}{c}\text { Degree of difference \% } \\
\text { GC } \\
\text { with reference strain } \\
\text { NCTC } 10373(\%)\end{array}$ \\
\hline $1 \mathrm{Sub}$ & 54.0 & 1.0 \\
\hline $2 \mathrm{Sub}$ & 54.5 & 0.5 \\
\hline $\mathrm{L}$ & 58.0 & 1.6 \\
\hline $6 \mathrm{~L}$ & 52.1 & 3.1 \\
\hline $10 \mathrm{~L}$ & 54.1 & 0.9 \\
\hline $\mathrm{Ch}$ & 56.0 & 1.0 \\
\hline $\mathrm{Ch}$ & 55.8 & 0.8 \\
\hline $8 \mathrm{~B}$ & 57.0 & 2.0 \\
\hline $10 \mathrm{~B}$ & 56.2 & 1.2 \\
\hline $12 \mathrm{Ch}$ & 57.8 & 2.8 \\
\hline
\end{tabular}

It was found that the isolated cultures of the causative agent of urocystitis and pyelonephritis in pigs had an average content of $55.5 \mathrm{~mole} \% \mathrm{G}+\mathrm{C}$. The variability range of the nucleotide composition of deoxyribonucleic acid was $\min =52.1 \mathrm{~mole} \% \mathrm{G}+\mathrm{C}$, $\max =58.0$ mole $\% \mathrm{G}+\mathrm{C}$.

\section{Conclusion}

In this way, the conducted comprehensive studies allow to state some conclusions, namely, the isolated cultures of the causative agent of urocystitis and pyelonephritis in pigs have a number of both constant and variable phenotypic characteristics. Thus, in all cases, the isolated A. suis microorganisms are catalase inactive, do not form indole, do not decompose mannose, have weakly expressed proteolytic activity and do not cause hemolysis, and at the same time have high urease activity. At the same time, A. suis cultures do not ferment xylose in $99.2 \%$ of cases, ribose - in $98 \%$, and maltose - in $96 \%$. The GC-pairs content in the DNA of cultures isolated from pigs in the farms of the Omsk region averaged $55.5 \mathrm{~mole} \%$. The study of the gene encoding $16 \mathrm{~S}$ rRNA by restriction analysis revealed no differences in PCR products, which indicates a high degree of genetic relation of the isolated Actinobaculum suis cultures. 


\section{References}

1. A. Krasikov, V. Vlasenko, V. Pleshakova, J. Alekseeva, Dynamics of immunological parameters of cattle infected with leukemia virus and correction with botulin-based medication, The fifth technological order: prospects for the development and modernization of the Russian agroindastrial sector, 393, 25-28 (2019)

2. T. Gerunov, M. Gonokhova, T. Boyko, V. Gerunov, Pathomorphogical changes in the organs and tissues of animals during acute intoxication with neonicotinoids containing nitrozo or cyano grup, Proceedings of the international scientific conference. The fifth technological order: prospects for the development and modernization of the Russian agroindustrial sector, 393, 421-426 (2019)

3. E. Yudina, S. Konovalova, Development Issues and Prospects of milk proceedings, Enterprises proceedings of the international scientific conference The fifth technological order: prospects for the development and modernization of the Russian agroindastrial sector, 393,436-439 (2019)

4. V.I. Pleshakova, T. I. Lorengel, Bacteriological diagnosis of urocystitis and pyelonephritis sows Omsk scientific Bulletin, 7(43), 171-173 (2006)

5. H. Berner, Veterinarian Umsch, 36, 162-171 (1981)

6. F. Madec, Pig News and Inform, 89-93 (1984)

7. S.A. Dee, Vet.Med, 86, 231-238 (1991)

8. G.J. Dagnall, Med.Lab.Sei, 40, 199 (1983)

9. J. Carr, J.R. Walton, Investigation of the pathogenetic properties of Eubacterium suis (Corynebacterium suis), (In: 11 th Congr.Int. Pig Vet.Soc., Lausanne, Proc.178, 1990)

10. J.L. Larsen, Nord. Veterinaermed, 22, 422-431 (1970)

11. M.A. Soltys, F.R. Spratling, School of Veterinari Medicine, University of Cambridge. The Veterinary record, 69, 575-582 (1957)

12. J.E.T Jones, Cystitis and pyelonephritis in sows caused by Corynebacterium suis: epidemiological considerations (In:Soc.Vet.Prev. Med., Solihull, Proc., 1987)

13. B. Aalvik, Nord Veterinaemed, 20, 319-320, (1968)

14. P. Lawson, E. Falsen, E. Akervall, Int. Syst.Bacteriol, 47(3), 899-903 (1997)

15. W. Ludwig, G. Kirchhof, M. Weizenegger, N. Weiss, International Journal of Systematic Bacteriology, 161-165 (1992) 